\title{
Video Article \\ Proteomic Sample Preparation from Formalin Fixed and Paraffin Embedded Tissue
}

\author{
Jacek R. Wiśniewski ${ }^{1}$ \\ ${ }^{1}$ Department of Proteomics and Signal Transduction, Max Planck Institute of Biochemistry
}

Correspondence to: Jacek R. Wiśniewski at jwisniew@biochem.mpg.de

URL: https://www.jove.com/video/50589

DOI: doi:10.3791/50589

Keywords: Chemistry, Issue 79, Clinical Chemistry Tests, Proteomics, Proteomics, Proteomics, analytical chemistry, Formalin fixed and paraffin embedded (FFPE), sample preparation, proteomics, filter aided sample preparation (FASP), clinical proteomics; microdissection, SAX-fractionation

Date Published: 9/2/2013

Citation: Wiśniewski, J.R. Proteomic Sample Preparation from Formalin Fixed and Paraffin Embedded Tissue. J. Vis. Exp. (79), e50589, doi:10.3791/50589 (2013).

\section{Abstract}

Preserved clinical material is a unique source for proteomic investigation of human disorders. Here we describe an optimized protocol allowing large scale quantitative analysis of formalin fixed and paraffin embedded (FFPE) tissue. The procedure comprises four distinct steps. The first one is the preparation of sections from the FFPE material and microdissection of cells of interest. In the second step the isolated cells are lysed and processed using 'filter aided sample preparation' (FASP) technique. In this step, proteins are depleted from reagents used for the sample lysis and are digested in two-steps using endoproteinase LysC and trypsin.

After each digestion, the peptides are collected in separate fractions and their content is determined using a highly sensitive fluorescence measurement. Finally, the peptides are fractionated on 'pipette-tip' microcolumns. The LysC-peptides are separated into 4 fractions whereas the tryptic peptides are separated into 2 fractions. In this way prepared samples allow analysis of proteomes from minute amounts of material to a depth of 10,000 proteins. Thus, the described workflow is a powerful technique for studying diseases in a system-wide-fashion as well as for identification of potential biomarkers and drug targets.

\section{Video Link}

The video component of this article can be found at https://www.jove.com/video/50589/

\section{Introduction}

Fixing with paraformaldehyde and embedding in paraffin (FFPE) is the standard method for preservation and pathomorphologic investigation of clinical tissue material. Microscopy of the preserved tissue allows identification of disease related morphological changes, and detection and scoring of occurrence of disease related antigens. Since typically only a small portion of the FFPE sample is used in these analyses, large amounts of clinical material remain archived and can be exploited in other types of studies.

During the recent decade the research in life sciences has been strengthened by the powerful proteomic technology. This technology allows identification and quantitation of thousands of proteins in complex protein mixtures. An important feature of the technology is that only minute amounts of material are needed for large scale analyses. Recent proteomic studies demonstrated that proteins can be studied on a scale of nearly complete proteomes ${ }^{1,2}$. This type of investigations enables system wide insights in the composition of the cells and identification of groups of proteins occurring at anomalous levels in diseases. Whereas these studies required large mass spectrometric capacities, the recent work has demonstrated that similar extent of identification can be achieved within a single day of measurement ${ }^{3}$.

The requirement of relatively low sample amount and the wide availability of the preserved clinical samples tempted us and others to develop methods enabling proteomic exploration of the FFPE material (for a review see ${ }^{4}$ ). Taking advantage of the reversibility of the formalin fixation under a heat treatment we have developed a protocol allowing quantitative extraction of proteins from the fixed tissue ${ }^{5}$. We have shown that the fixation procedure preserves not only proteins, but also at least some posttranslational modifications. Phosphorylation and $\mathrm{N}$-glycosylation can be studied using the FFPE samples ${ }^{5}$, however a prerequisite for it is a thoroughly controlled tissue collection, storage and fixation conditions. Next, we have optimized the method for a laser capture microdissection of the fixed tissue and for the proteomic reactor based sample processing 6,7 . A large scale study on colorectal cancer allowed quantitative analysis of 7,500 proteins from microdissected from clinical material ${ }^{8}$. Finally, we have improved the way of exploration of microdissected material by applying consecutive-two step protein digestion protocol ${ }^{9}$. In comparison to the common digestion strategies using single enzyme, this procedure enables generation of more sequence-unique peptides and, consequently, results in a higher depth of protein identification. Analysis of microdissected human colonic adenoma allowed proteomic analysis to a depth of 9,500 proteins per sample ${ }^{3}$. In this study we mapped 55,000 peptides per sample allowing identification of $88-89 \%$ proteins with at least 2 peptides. Here we present an optimized protocol for preparation of samples from the FFPE material for LC-MS/MS analysis. This protocol comprises preparation of tissue slices for microdissection, lysis of the isolated cells, and sample processing in a reactor format (FASP) ${ }^{6}$. Then we describe a spectrofluorometric determination of peptide yields; a task with high importance for optimal peptide identification by the mass spectrometry. Finally, we demonstrate a strong anion exchanger (SAX)-based separation technique for peptide fractionation. In this method both 
the peptide separation and final cleanup are carried out using pipette-tip microcolumns ${ }^{10,11}$. This step is optional but is recommended in studies in which more than few microgram peptides can be obtained from a single sample. The SAX-fractionation can substantially increase the number and dynamic range of identifications and extend the protein sequence coverage ${ }^{3,10}$.

\section{Protocol}

\section{Instrumentation Required}

1. Preparation of tissue slices for microdissection and sample lysis

1. Thermoblock set to $99{ }^{\circ} \mathrm{C}$ or a bath with boiling water.

2. Laser operating microdissector (for example PALM, Zeiss, Göttingen, Germany).

3. Thermostated bench-top centrifuge, temperature set to $20^{\circ} \mathrm{C}$.

4. Wet chamber (box) with a rack for Eppendorf-type disposal tubes.

5. Incubator set to $37^{\circ} \mathrm{C}$.

6. Spectrofluorometer enabling measuring of fluorescence excited near UV light with micro-scale quartz cuvettes (0.1-0.2 ml).

\section{Solutions}

1. 'Tissue Lysis Buffer' (TLB): of $0.1 \mathrm{M}$ Tris-HCl, $\mathrm{pH} 8.0,0.1 \mathrm{M} \mathrm{DTT}, 0.5 \%$ (w/v) polyethylene glycol 20,000 and $4 \%$ SDS

2. UA solution: $8 \mathrm{M}$ urea in $0.1 \mathrm{M}$ Tris- $\mathrm{HCl} \mathrm{pH} \mathrm{8.5}$. Prepare $1 \mathrm{ml}$ per 1 sample. UA and IAA solutions have to be prepared freshly and used within a day.

3. IAA solution: $0.05 \mathrm{M}$ iodoacetamide in UA. Prepare $0.1 \mathrm{ml}$ per 1 sample.

4. Endoproteinase Lys-C, stock solution.

5. Trypsin, stock solution.

6. 'Digestion buffer' (DB): $0.05 \mathrm{M}$ Tris- $\mathrm{HCl} \mathrm{pH}$ 8.5. Prepare $0.25 \mathrm{ml}$ per 1 sample.

7. 'Tryptophan Standard Solution': $0.1 \mu \mathrm{g}$ tryptophan in $1 \mathrm{ml}$ deionized water.

8. 'Assay buffer B' (ABB): $10 \mathrm{mM}$ Tris-HCl, pH 7.6.

9. Stock solution of Britton \& Robinson Universal Buffer (BRUB). Prepare a solution containing $0.1 \mathrm{M} \mathrm{CH}_{3} \mathrm{COOH}, 0.1 \mathrm{M} \mathrm{H}{ }_{3} \mathrm{PO}$, and $0.1 \mathrm{M}$ $\mathrm{H}_{3} \mathrm{BO}_{3}$ and adjust with $1 \mathrm{M} \mathrm{NaOH}$ to the required $\mathrm{pH}(2,4,5,6$, and 11). Before use dilute 5-fold with deionized water.

10. Buffer $\mathrm{A}: 1 \%(\mathrm{v} / \mathrm{v}) \mathrm{CH}_{3} \mathrm{COOH}$ in deionized water.

11. Buffer $\mathrm{B}: 60 \%(\mathrm{v} / \mathrm{v}) \mathrm{CH}_{3} \mathrm{CN}, 1 \%(\mathrm{v} / \mathrm{v}) \mathrm{CH} 3 \mathrm{COOH}$ in deionized water.

\section{Pipette Tip and 'StageTip' Columns}

1. Prepare SAX tip-column by stacking 6 layers of Empore- Anion membrane in a common $0.2 \mathrm{ml}$ pipette tip. Make two SAX-tip columns per sample.

2. Prepare 'StageTip' by stacking 3 layers of Empore- $\mathrm{C}_{18}$ in a $0.2 \mathrm{ml}$ pipette tip. Make 6 Stage tips for each sample.

\section{Preparation of Tissue Slices for Microdissection and Sample Lysis}

1. Cut sections with a microtome (the thickness of the slices depends on the sample. Usually microdissection works well with slices of 7-10 $\mu \mathrm{m}$ ).

2. Irradiate membrane slides (MembraneSlide 1.0 PEN) with UV-light for $45 \mathrm{~min}$.

3. Mount the sections on the irradiate membrane slides and dry at $37^{\circ} \mathrm{C}$ for $2 \mathrm{hr}$.

4. Deparaffinize the mounted sections by two successive incubations in xylene for 2.5 min and 1.5 min. Rehydrate the sections by consecutive incubations in absolute ethanol, $70 \%$ ethanol, and water, each for $1 \mathrm{~min}$.

5. Stain the sections with Mayer's hematoxylin for $20 \mathrm{sec}$, rinse with water for $1 \mathrm{~min}$, and air-dry.

6. Collect the cell population of interest using laser capture microdissection system. When using the PALM instrument (Zeiss) collect the samples in the adhesive caps (Adhesive CAP 200).

7. Pipette an aliquot of the TLB over the microdissected material in the cap. Close the tube and collect the suspension by a short centrifugation. Lyse the microdissected tissue at $99^{\circ} \mathrm{C}$ in a heating block with agitation $(600 \mathrm{rpm})$ for $1 \mathrm{hr}$. Use $3 \mu \mathrm{l}$ of buffer for $10 \mathrm{nl}$ of the microdissected tissue.

8. Clarify the crude extract by centrifugation at $16,000 \times \mathrm{g}$ at $18^{\circ} \mathrm{C}$ for $10 \mathrm{~min}$. The lysate can be stored frozen at $-20^{\circ} \mathrm{C}$.

\section{Sample Processing}

1. Mix up to $50 \mu \mathrm{l}$ of the clarified lysate with $200 \mu \mathrm{l}$ of UA in the ultrafiltration units (Forensic $30 \mathrm{k}$ ) and centrifuge at $14,000 \times \mathrm{g}$ until less than 10 $\mu \mathrm{l}$ of the solution will remain in the filter. Usually this step requires 10-15 min centrifugation.

2. Pipette $200 \mu$ l of UA to the ultrafiltration unit and centrifuge as in 5.1. Empty the collection tube.

3. Pipette $50 \mu \mathrm{l}$ of IAA solution and $\mathrm{mix}$ at $600 \mathrm{rpm}$ in a thermo-mixer for $1 \mathrm{~min}$ and centrifuge as in 5.1 .

4. Pipette $100 \mu \mathrm{l}$ of UA to the ultrafiltration unit and centrifuge as in 5.1. Repeat this step twice

5. Pipette $100 \mu \mathrm{l}$ of the DB to the ultrafiltration unit and centrifuge at in 5.1. Repeat this step twice.

6. Transfer the filtration units to new collection tubes. Pipette $40 \mu \mathrm{I} \mathrm{DB}$ with endoproteinase Lys-C (proteinase to total protein ratio of $1: 100$ ) and mix at $600 \mathrm{rpm}$ in thermo-mixer for $1 \mathrm{~min}$. Incubate the units in a wet chamber at $37^{\circ} \mathrm{C}$ for $18 \mathrm{hr}$.

7. Centrifuge the filter units at $14,000 \times \mathrm{g}$ such as in 5.1 . 
8. Pipette $160 \mu \mathrm{l}$ of deionized water and centrifuge the filter units as in 5.1 . The pooled flow-through ( 5.7 and 5.8 steps) contains the peptides released by endoproteinase Lys $\mathrm{C}$.

9. Transfer the ultrafiltration unit to a new collection tube. Add $40 \mu \mathrm{LB}$ with trypsin (enzyme to protein ratio 1:100) and mix at $600 \mathrm{rpm}$ in thermo-mixer for $1 \mathrm{~min}$. Incubate the units in a wet chamber at $37^{\circ} \mathrm{C}$ for $4 \mathrm{hr}$. Repeat steps 5.7 and 5.8 to collect the tryptic peptides.

\section{Quantitation of the Peptides}

1. The measurement of the peptide content in the protein digests can be performed in diluted buffer because the tryptophan residues are well accessible to the solvent.

2. Pipette $0.2 \mathrm{ml}$ of $A B B$ into the quartz cuvette and record the emission spectrum for 'blank'.

3. Prepare calibration curve using $0.1 \mu \mathrm{g}$ steps by adding $1 \mu \mathrm{l}$ aliquots of TSS to the cuvette and gentle mix with the ABB.

4. Clean the cuvette.

5. Pipette the sample and record the spectrum (sample).

6. Calculate of the protein and peptide concentration

Since $0.1 \mu \mathrm{g}$ tryptophan corresponds to about $9 \mu \mathrm{g}$ protein (based on the average $1.1 \%$ of the tryptophan content in protein mixtures obtained by lysing human cells) the protein content of the in the sample or peptide content in the digest equals:

Peptide content $(\mu g)=9 \times \frac{F(\text { Sample })-F(\text { Blank })}{F(\text { Standard } 1)-F(\text { Blank })}$ where $F($ Standard 1$)$ is the fluorescence of $0.1 \mu \mathrm{g}$ tryptophan standard. The peptide content allows calculation of the yield of the digestion procedure and provides essential information for following peptide fractionation and mass spectrometric analysis.

\section{Fractionation of the Lys-C and Tryptic Peptides}

1. Dilute the peptide solutions obtained by digestions with Lys-C and trypsin with $0.2 \mathrm{ml}$ of BRUB pH 11 and pH 5 , respectively.

2. Assemble the SAX tip-column in the centrifugal tube adapter lid.

3. Wash and equilibrate the SAX tip-column successively with $0.1 \mathrm{ml}$ of methanol, $0.1 \mathrm{ml}$ of $1 \mathrm{M} \mathrm{NaOH}$, and 3 times with $0.1 \mathrm{ml}$ of BRUB, pH 11 for the separation of Lys-C peptides and with BRUB, $\mathrm{pH} 5$ for the tryptic peptides. The flow of the solutions through the column material is facilitated by centrifugation at $4,000 \times \mathrm{g}$.

4. Wash and equilibrate the $\mathrm{C}_{18^{-}}$'StageTips' subsequently with, $0.05 \mathrm{ml}$ of methanol, then with $0.05 \mathrm{ml}$ of Buffer B and with $0.05 \mathrm{ml}$ of Buffer $\mathrm{A}$. Prepare six $\mathrm{C}_{18}$-'StageTips'; 4 for the separation of the Lys-C peptides and two for the separation of the tryptic peptides.

5. Assemble the SAX Tip-column in the $\mathrm{C}_{18}$-'StageTip'. Load the sample solutions into the equilibrated SAX Tip-columns and centrifuge the columns at $5,000 \times \mathrm{g}$ for $3 \mathrm{~min}$.

6. Equilibrate the 'pipette-tip' columns with $0.1 \mathrm{ml}$ of the BRUB, $\mathrm{pH} 11$ or $\mathrm{pH}$, respectively to the Lyc-C and tryptic samples. Facilitate the flow by centrifugation at $5,000 \times \mathrm{g}$.

7. Transfer the SAX Tip-column to the next $\mathrm{C}_{18}$-'StageTip'.

8. Continue eluting of the peptides with BRUB pH 6,4 , and 2 for the Lys $\mathrm{C}$ sample and with BRUB pH 2 for the sample with tryptic peptides. For each elution step use a separate $\mathrm{C}_{18}$-'StageTip'.

9. Wash the $\mathrm{C}_{18^{-}}$'StageTips' with $0.05 \mathrm{ml}$ of Buffer A.

10. Elute fractions with $0.05 \mathrm{ml}$ of Buffer B into vials used directly for injection of the samples into LC system assembled with a mass spectrometer.

\section{Representative Results}

Proteomic analysis of FFPE material requires robust and reproducible methods for sample preparation. In this publication we demonstrate a protocol allowing effective isolation of cell populations from the fixed material and their chemical processing resulting in high purity peptide fractions that can be directly used for LC-MS/MS analysis. The procedure consist of four distinct parts: (1) preparation of sections of the FFPE samples and its microdissection, (2) lysis of the sample and processing of the proteins using MED FASP approach, and (3) quantitation and (4) fractionation of the peptides (Figure 1).

In the first step of the procedure the FFPE samples are sectioned with a microtome and mounted on membrane covered slides. To increase the adhesion between the tissue sections and the surface of the slide membrane is necessary to irradiate the slide surface with UV light. For this purpose we use the light of a UV lamp integrated in to a cell culture bench. After this step the slides are subjected to washes allowing removal of paraffin and rehydration of the samples. The rehydrated material is stained with hematoxilin for a short time. This results in a weak staining of the sample but is sufficient for visualization of the sample areas for microdissection. The staining process has to be stopped rapidly by rinsing of the slides with an excess of water. A prolonged staining of the sample results in poor peptide yields.

In the next step the dried sections are subjected to microdissection. The selection of the tissue areas of individual cells depends on the type of investigation and always requires specific knowledge in histology or pathomorphology. The laser-released material is collected on adhesive surfaces of the sample collection tubes. Since the binding capacity of the adhesive material is limited it is necessary to transfer the microdissected material from the lid to the tube after dissection of every 3-5 $\mathrm{mm}^{2}$ of the sample. This can be easily done by pipetting of a few microliters of the tissue lysis buffer (LTB) onto the lid and a short spinning of the tube in a centrifuge. After the sample is collected in the bottom of the tube, the microdissection and the sample collection on the lid can be continued. Once the whole sample is collected the tubes have to be additionally stoppered with Parafilm and incubated in a water bath at about $99^{\circ} \mathrm{C}$ with continuous agitation for $1 \mathrm{hr}$. Since during the incubation water condenses on the lid, every $15 \mathrm{~min}$ the tubes have to be shortly centrifuged to collect back the water in the tube cone.

To obtain peptides the tissue lysates are processed using the MED-FASP approach. In this method the lysed proteins are depleted from the detergent and other low molecular weight substances, carboamidomethylated at cysteinyl residues, and then consecutively digested with two enzymes differing in their cleavage specificities: endoproteinase LysC and trypsin. After each digestion step the peptides are collected into 
separate fractions. In this part of the sample preparation it is important to continue each of the centrifugation steps until more than $95 \%$ of the solution initially loaded into the filtration unit passed the membrane. Analyzing colonic tissue and its cancer we observed that this procedure yields $3-6 \mu \mathrm{g}$ of peptide per $100 \mathrm{nl}\left(100 \mu \mathrm{g}\right.$ tissue or $10 \mathrm{~mm}^{2}$ of a $10 \mu \mathrm{m}$ section) of the microdissected material (Table 1). Since the extractable total protein from tissues is about $10 \%$ of the tissue weight the extraction and digestion procedures together result in a $30-60 \%$ yield in the respect to the original fresh tissue. These values reflect losses of the protein during dehydration and staining, microdissection, and retention of the portion of the undigested sample in the ultrafiltration units. Because extraction and processing of non-microdissected FFPE tissue resulted in protein-to-peptide-conversion yield of $75 \%{ }^{5}$ it is likely that the critical sample losses occur during the steps before MED-FASP. An important feature of the peptide mixtures obtained by this procedure is their high purity which facilitates further fractionation peptide fractionation process and the mass spectrometry identification. This has recently been shown by an analysis of colorectal adenoma samples ${ }^{3}$. In that study about $40 \%$ of the MS/MS events led to identification of peptides from the FFPE tissue and resulted in mapping of up to 17,000 peptides per single LCMS/MS run. Higher identification rates have been achieved only in analysis of frozen in vitro cultured cells ${ }^{3}$.

In the two last parts of the whole procedure the isolated peptides are quantified and fractionated on pipette-tip microcolumns. Since the amounts of peptide isolated from the microdissected tissue are below $10 \mu \mathrm{g}$ they cannot be quantified using commonly used dye-based protein assays. Also the $A_{280} \mathrm{UV}$-spectral measurements at these concentrations are not useful due to light scattering effect. In contrast, measurements of fluorescence of the tryptophan residues offer a reliable way for determination of the peptide content.

Recently we have shown that up to 5,000 proteins from cultured cells can be identified in a 'single shot' 4 hr LC-MS/MS analysis ${ }^{7}$. However this type of analysis applied to native tissues seldom allows identification of more than 3,000 thousand proteins. To increase of the depth of the analysis the peptides generated in MED FASP have to be pre-fractionated before LC-MS/MS. The SAX based micro-column separation is a simple and efficient fractionation method ${ }^{10}$. It has already been applied in several proteomic studies including those analyzing fixed tissue , $^{5,9}$ The SAX-'pipette-tip' microcolumns, and the $\mathrm{C}_{18}$-'StageTip' desalting columns ${ }^{9}$ are easy to prepare. The columns are assembled by stacking of plugs, cut from the SAX or $\mathrm{C}_{18}$ membranes, in a $200 \mu$ pipette tip ${ }^{11}$. Examples of the analysis of the SAX-fractionated FFPE samples are shown in Table 1.

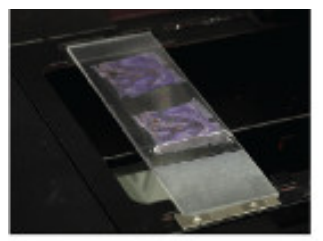

\section{Sample microdissection and lysis}

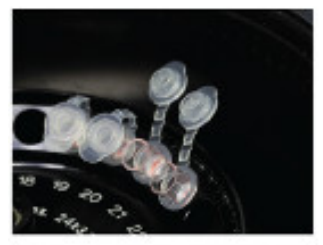

\section{MED-FASP-processing}

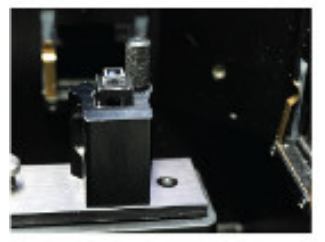

\section{Determination of peptide concentration}

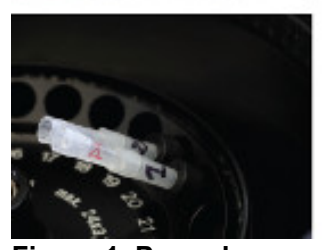

\section{SAX fractionation}

Figure 1. Procedure overview. The procedure consist of four distinct parts: (1) preparation of sections of the FFPE samples and its microdissection, (2) lysis of the sample and processing of the proteins using MED FASP approach, and (3) quantitation and (4) fractionation of the peptides.

\begin{tabular}{|c|c|c|c|c|c|}
\hline Sample & $\begin{array}{l}\text { Sample pre- } \\
\text { fractionation / } \\
\text { digestion }\end{array}$ & $\begin{array}{l}\text { Mass } \\
\text { spectrometer } \\
\text { used }\end{array}$ & $\begin{array}{l}\text { Peptide yield } \mathrm{ng} / \mathrm{nL} \\
\text { sample ( } \pm S D)\end{array}$ & $\begin{array}{l}\text { Unique Protein } \\
\text { Identifications Per } \\
\text { single sample }\end{array}$ & Reference \\
\hline \begin{tabular}{|l}
-Adenonocarcinoma \\
-Normal colonic mucosa
\end{tabular} & SAX/one enzyme & Orbitrap -Velos & $\begin{array}{l}36 \pm 11(n=8) \\
30 \pm 8(n=8)\end{array}$ & $\begin{array}{l}5,985 \pm 54 \\
5,868 \pm 110\end{array}$ & 8 \\
\hline -Colonic Adenoma & SAX/two enzymes & Q Exactive & $56 \pm 6.1(n=3)$ & $9,501 \pm 28$ & 3 \\
\hline
\end{tabular}

Table 1. Representative results of proteomic analyses of the FFPE microdissected tissue. 


\section{Discussion}

The ability to study the FFPE material by the proteomic technology to a depth comparable with nucleic acid sequencing and microarray techniques opens new perspectives in biomarker and drug target discovery. The described protocol enables characterization and quantitation of proteomes of populations of microdissected cells on a scale of 10,000 proteins. When using less of the microdissected material a smaller datasets can be generated, but perhaps in many cases those can also provide valuable clinical data. Thus, either the samples can be analyzed directly after the MED-FASP procedure or can be separated in less fractions. Since FASP procedure is compatible with any type of protease, other enzymes or their combination can be used from protein digestions ${ }^{6}$.

The quality of the FFPE material appears to be the most critical issue of the analysis. We have experienced that the fixed tissue of the same origin but coming from different clinics had distinct properties. Using tissue from one source we were able to generate peptides at high yields, whereas similar material from another clinic was almost useless. It is likely that the applied fixation and embedding procedures as well as storage conditions are the major factors affecting the properties of the clinical material ${ }^{12}$. Therefore it is advisable to test the properties of few samples before starting a larger project.

Many publications reported the use of the FFPE material in the past. However, the number of proteins identified in these studies never exceeded more than $1,000-2,000$ proteins ${ }^{4}$. Considering the size of the human cell specific proteomes comprising more than 10,000 proteins, such studies were able only to provide a very superficial picture, almost limited to highly abundant proteins involved in housekeeping functions. Our protocol enables efficient isolation of the clinical or biological material from preserved tissue and is optimized for lysis, protein processing and peptide prefractionation. As a consequence our sample preparation workflow, when coupled to the high-end mass spectrometry, allows insights into a nearly complete proteomes. Notable, this is possible at minute sample amount requirements.

The major advantage of using the preserved tissues is their relative wide availability. FFPE samples archived for many years and decades, and sometimes considered as useless, now, thanks to proteomic technology, appear as highly valuable material for clinical research. Whereas many diseases can be studied using fresh or frozen material investigation of FFPE material seems to be particularly important for studying rare diseases ${ }^{12}$, were collection of a representative set of sample takes usually a long time.

\section{Disclosures}

The author has nothing to disclose.

\section{Acknowledgements}

The author thanks Dr. Matthias Mann for the continuous support and Mrs. Katharina Zettl for demonstrating the method.

This work was supported by the Max-Planck Society for the Advancement of Science.

\section{References}

1. Nagaraj, N., et al. Deep proteome and transcriptome mapping of a human cancer cell line. Molecular systems biology. 7, 548 (2011).

2. Beck, M., et al. The quantitative proteome of a human cell line. Molecular systems biology. 7, 549 (2011).

3. Wisniewski, J.R., Dus, K., \& Mann, M. Proteomic workflow for analysis of archival formalin-fixed and paraffin-embedded clinical samples to a depth of 10000 proteins. Proteomics. Clin. Appl. 7(3-4), 225-33 (2013).

4. Magdeldin, S. \& Yamamoto, T. Toward deciphering proteomes of formalin-fixed paraffin-embedded (FFPE) tissues. Proteomics. 12(7), 1045-58 (2012).

5. Ostasiewicz, P., et al. Proteome, phosphoproteome, and N-glycoproteome are quantitatively preserved in formalin-fixed paraffin-embedded tissue and analyzable by high-resolution mass spectrometry. J. Proteome Res. 9(7), 3688-700 (2010).

6. Wisniewski, J.R., et al. Universal sample preparation method for proteome analysis. Nature methods. 6(5), 359-62 (2009).

7. Wisniewski, J.R., Ostasiewicz, P., \& Mann, M. High recovery FASP applied to the proteomic analysis of microdissected formalin fixed paraffin embedded cancer tissues retrieves known colon cancer markers. Journal of proteome research. 10(7), 3040-9 (2011).

8. Wisniewski, J.R., et al. Extensive quantitative remodeling of the proteome between normal colon tissue and adenocarcinoma. Mol. Syst. Biol. 8, 611 (2012).

9. Wisniewski, J.R. \& Mann, M. Consecutive proteolytic digestion in an enzyme reactor increases depth of proteomic and phosphoproteomic analysis. Anal. Chem. 84(6), 2631-7 (2012).

10. Wisniewski, J.R., Zougman, A., \& Mann, M. Combination of FASP and StageTip-based fractionation allows in-depth analysis of the hippocampal membrane proteome. J. Proteome Res. 8(12), 5674-8 (2009).

11. Rappsilber, J., Ishihama, Y., \& Mann, M. Stop and go extraction tips for matrix-assisted laser desorption/ionization, nanoelectrospray, and LC/ MS sample pretreatment in proteomics. Anal. Chem. 75(3), 663-70 (2003).

12. Thompson, S.M., et al. Impact of pre-analytical factors on the proteomic analysis of formalin-fixed paraffin-embedded tissue. Proteomics Clin. Appl. (2012). 